\title{
Reply to "On Cochlear Impedances and the Miscomputation of Power Gain" by Shera et al. J. Assoc. Re. Otolaryngol.
}

\author{
Tianying Ren, ${ }^{1}$ Wenxuan He, ${ }^{1}$ and Peter G. Gillespie ${ }^{1,2}$ \\ ${ }^{1}$ Oregon Hearing Research Center, Department of Otolaryngology and Head and Neck Surgery, Oregon Health Ev Science \\ University, 3181 SW Sam Jackson Park Road, NRC04, Portland, OR 97239, USA \\ ${ }^{2}$ Vollum Institute, Oregon Health E Science University, 3181 SW Sam Jackson Park Road, Portland, OR 97239, USA
}

Received: 19 August 2011; Accepted: 25 September 2011; Online publication: 21 October 2011

\begin{abstract}
Using a scanning laser interferometer, we recently measured the volume velocity of the basilar membrane vibration in the sensitive gerbil cochlea and estimated that the cochlear power gain is $\sim 100$ at low sound pressure levels (Ren et al., Nat Commun 2:216-223, 2011a). We thank Shera et al. for recognizing the technical challenges of our experiments and appreciating the beauty of our data in their comment (Shera et al., J Assoc Res Otolaryngol (in press), 2011). These authors argue that our analysis is inappropriate, invalidating our conclusion; moreover, they suggest that our finding of a power gain of $>1$ could arise from a passive structure or cochlea. While our analysis and interpretation remain to be verified, they are justified according to commonly accepted assumptions and theories in cochlear mechanics. Here, we also show that the mathematical demonstration of a power gain of $>1$ in a passive cochlea by Shera et al. is inconsistent with our data, which show that the volume velocity and power gain decrease and become $<1$ as the sound level increases.
\end{abstract}

Keywords: Cochlea, basilar membrane vibration, cochler amplifier, laser interferometer, traveling wave

Shera et al. (2011) criticized our study for not measuring impedance of the cochlear partition, or

Correspondence to: Tianying Ren · Oregon Hearing Research Center, Department of Otolaryngology and Head and Neck Surgery · Oregon Health \& Science University - 3181 SW Sam Jackson Park Road, NRC04, Portland, OR 97239, USA. rent@ohsu.edu pressure across the partition, to quantify the cochlear power gain. Although the cochlear power can be quantified as the product of velocity and pressure, neither the volume velocity nor distributed pressure have been measured previously. To determine whether the cochlea generates power to amplify soft sounds, numerous studies have been conducted using different approaches. For example, de Boer and Nuttall (1999) calculated the organ of Corti impedance by coupling frequency-response data to a cochlear model. In their analysis, the frequency response of the basilar membrane measured at a single location was converted into a spatial response using the cochlear map. Negative resistance of the cochlear partition was detected at a location basal to the response peak. To confirm this important finding, Olson (2001) quantified the organ of Corti resistance by directly measuring intracochlear pressure. She found that the pressure near the basilar membrane was as tuned and nonlinear as the basilar membrane vibration, which resulted in a relatively untuned and linear impedance of the organ of Corti. Since the result could not decisively determine whether the cochlea generates power, Olson suggested that negative resistance might be better measured via a more detailed map of the scala tympani pressure using a smaller pressure sensor. Due to technical challenges, detailed mapping of the intracochlear pressure near the cochlear partition remains to be conducted at the time this letter was written.

By developing a scanning laser interferometer, we measured the volume velocity of the basilar membrane vibration, and, by using commonly accepted assumptions in cochlear mechanics, estimated the relevant power. Most cochlear mathematical models 
(Peterson and Bogert 1950; Zweig 1976; Zwislocki 1953) present the cochlear traveling wave using a series of independent sections along the cochlea and assume that acoustic energy is transmitted through the cochlear fluid (Wever et al. 1954). In one-dimensional cochlear models, it is assumed that the basilar membrane vibrates uniformly over the entire width of the cochlear partition (Puria and Steele 2008) and that cochlear fluid moves in a longitudinal direction (de Boer 1996). This assumption, however, is contradicted by the physical requirement that the fluid must also move up and down with the basilar membrane. To avoid the paradox, it is further assumed that the vertical and longitudinal components of the fluid velocity change over the height of the cochlear duct. When longitudinal coupling in the organ of Corti is neglected, the traveling wave could be presented as a series of independent oscillators along the cochlear partition. In fact, the constant organ of Corti mass and frequency-dependent effective mass of the cochlear fluid were used to calculate the active force of the organ of Corti complex (Olson 2004). Olson also demonstrated two types of intracochlear pressure waves: one near the basilar membrane related to the traveling wave, and the other uniformly distributed in the entire cochlea associated with compression wave (Olson 2004). Since each section of the cochlear partition and nearby fluid vibrates in the transverse direction, which cannot transmit energy in the longitudinal direction, the compression wave in the cochlear fluid likely is responsible for the longitudinal power transmission. Based on this assumption, sound power in the cochlear fluid can be estimated using fluid velocity and impedance, and cochlear power gain can be estimated as the ratio of power in the fluid near the stapes to that at the best-frequency location.

Since the speed of sound $(c)$ is related to impedance, $c$ in the cochlear fluid near the stapes can be calculated from the cochlear input impedance $\left(Z_{c}\right)$. Based on measured $Z_{c}$ in cat (Lynch et al. 1982) and human (Aibara et al. 2001), Puria and Steele (2008) found that the speed of sound in the cochlear fluid near the stapes is $\sim 15$ times lower than the speed of sound in water, which is consistent with the speed of the cochlear slow wave. As the authors pointed out, it is the flexibility of the cochlear partition that slows the wave speed. Thus, the slow sound speed is dominated likely by the slow transverse component of the fluid velocity and may not necessarily exclude the existence of a fast longitudinal component.

Shera et al. also argue that our power measurements have incorrect dimensions. Because of the dispersive nature of the cochlear traveling wave (Lighthill 1981), volume velocity must be measured in a defined time or area that allows comparison between the measurement near the stapes and that at the best-frequency location. We measured the volume velocity over a half-wavelength area centered at the best-frequency location. We chose a half-wavelength area rather than a random length because the vibration over a half-wavelength area is in phase and the volume displacement over this area can be compared to that at the stapes. The sound energy passing through the in-phase-vibration area in $1 \mathrm{~s}\left(I_{b f}\right)$ was calculated according to $I_{b f}=\left(V_{b f V}\right)^{2} \rho c$, where $V_{b f V}$ is the volume velocity $\left(V_{b f V}=2 f \pi V_{b f+}, f\right.$, and $V_{b f+}$ are frequency and the volume displacement), $\rho$ is the density of the cochlear fluid and $c$ is the speed of sound in water. Shera et al. are correct that in the standard equation $I=\rho c V^{2}, I$ is the acoustic intensity (time-averaged power per unit area), and $V$ is the point velocity. $V$ was replaced by the volume velocity $V_{b f V}$ in our analysis because the volume velocity over a half-wavelength area at the best-frequency place is comparable to the stapes volume velocity. This results in a dimension of power $\mathrm{x}$ area ([power][area]= $\left(M L^{2} / T^{3}\right) L^{2}$, where $M, L$, and $T$ represent the dimensions of mass, length, and time) as Shera et al. pointed out. Since $I_{b f}$ in our analysis is the energy passing through the in-phase-vibration area in $1 \mathrm{~s}$, the above dimension should be divided by $L^{2}$ and multiplied by $T$, resulting in a dimension of energy ([energy] $=M L^{2} / T^{2}$ ). Therefore, $I_{b f}$ is in joules, a unit of energy.

Another criticism by Shera et al. is about the area over which we measured the basilar membrane volume velocity. It is true that the total instantaneous volume displacement of the basilar membrane, integrated along the cochlear length, should be equal to the volume displacement of the stapes when the compressibility of the cochlear fluid and the fluid flow through the helicotrema are negligible. Shera et al. argue that this integrated displacement, measured as the stapes volume displacement in our experiment, must be smaller than the volume displacement over a half-wavelength area centered at the best-frequency location. Therefore, our finding of the cochlear power gain of $>1$ can exist in a passive cochlea. Although the volume displacement at a given halfwavelength region can be canceled by that at adjacent areas as shown by Fig. 1C (Ren et al. 2011a), whether the integrated volume displacement along the cochlear length is smaller than that over a half-wavelength area centered at the best-frequency location remains to be demonstrated. Our data show that the volumevelocity gain decreases with the stimulus level from $>10$ at $10 \mathrm{~dB}$ SPL to $<1$ at 80 and $90 \mathrm{~dB}$ SPL (red solid line in Fig. 5D). Therefore, our experiment result is not consistent with the argument by Shera et al.

One may find that that the gains in Fig. 5D (Ren et al. 2011a) apparently are a function of the length of the cochlear partition used for the volume displace- 
ment or velocity measurement. The gain increases from unity to $\sim 100$ at $10 \mathrm{~dB}$ SPL as the length for integrating volume velocity decreases from the entire cochlear length to the minimum, i.e., the single point. Since the peak response at the best-frequency location results from the cochlear amplifier, the volume velocity obtained from the entire cochlear length provides no specific information on the cochlear amplifier. Both the single-point velocity and the volume velocity over a half-wavelength area centered at the best-frequency location can be used to study the cochlear amplification. The single-point data has revealed the remarkable sensitivity, sharp tuning, and compressive nonlinearity of the cochlear partition vibration (Cooper and Rhode 1992; Nuttall et al. 1991; Ren 2002; Rhode 1971; Ruggero and Rich 1991; Russell and Nilsen 1997), which are the experimental basis of the cochlear-amplifier theory. The singlepoint velocity data provide limited or no information, however, on the power associated with the cochlear partition vibration. To calculate power near the bestfrequency location, we measured the volume velocity of the cochlear partition vibration. Since the cochlear amplifier locates at a small region basal to the bestfrequency location (de Boer and Nuttall 1999; Ren et al. 2011b), we measured the stapes volume velocity as the input and the volume velocity centered at the best-frequency location as the output to determine the volume-velocity gain. The volume velocity measured from a region smaller or larger than the halfwavelength area cannot be used to calculate the volume-velocity gain because they are not comparable to the stapes volume velocity.

To demonstrate that our finding of the cochlear power gain $\left(G_{\mathrm{e}}\right)$ of $>1$ can exist in a completely passive structure, Shera et al. computed $G_{\mathrm{e}}$ using a onedimensional cochlear model (Zweig 1976) and showed $G_{\mathrm{e}}$ is $>1$ under passive conditions. According to $G_{\mathrm{e}}=\left(G_{\mathrm{vol}}\right)^{2}$, a $G_{\mathrm{e}}$ of $>20$ in their Fig. 1 indicates a volume-velocity gain $\left(G_{\mathrm{vol}}\right)$ of $>4.47$. This is not consistent with our data which show that both $G_{\mathrm{vol}}$ and $G_{\mathrm{e}}$ are $<1$ at 80 and $90 \mathrm{~dB}$ SPL (blue and red lines in Fig. 5D, Ren et al. 2011a), a passive cochlear condition (Robles and Ruggero 2001).

Shera et al. also compared the cochlear volumevelocity gain to that in passive acoustic transformers or in the middle ear (Puria 2003) and argued that the volume-velocity gain cannot be used to estimate the power gain. However, when the volume velocity is measured at different locations in the same media with identical impedances, the volume-velocity gain can indeed be used to estimate the power gain. Neither an acoustic transformer nor the middle ear is comparable to the cochlea because they provide impedance matching between two media; by contrast, the basilar membranes at the base and at the best- frequency location are in the same cochlear fluid. The cochlear amplifier gain has been estimated from nonlinear compression of the basilar membrane vibration measured from a single location (Robles and Ruggero 2001). Figure 5D in our paper shows that both the volume-velocity and power gain decrease with the sound level as much as $>20$ and $>40 \mathrm{~dB}$, respectively. The compression of $>40 \mathrm{~dB}$ of the power gain is consistent with the result that the cochlear power gain is $>100$ at low sound levels, where the cochlear amplifier may dominate the cochlear mechanical response.

The volume velocity can also be used to study cochlear mechanisms using alternative analyses. Dong and Olson (2008) demonstrated $\sim 20-\mathrm{dB}$ nonlinear compression of intracochlear pressure measured from a single location near the basilar membrane. If this pressure data can be used as an estimate of the averaged pressure over the in-phase-vibration area and the $\sim 20-\mathrm{dB}$ compression is considered as the cochlear pressure gain, the volume-velocity gain of $\sim 10$ shown by our data indicates a power gain of $\sim 100$. Thus, our method apparently reliably indicates the cochlear amplifier gain.

In summary, although our analysis remains to be further verified, our experimental data demonstrate that the volume-velocity gain is $\sim 100$ times smaller than the point velocity gain measured from a single cochlear location. These results extend our view of cochlear mechanical response from a single point into a more realistic two-dimensional area on the cochlear partition. Hopefully, ongoing discussions of these new data will facilitate greater understanding of hearing mechanisms.

\section{ACKNOWLEDGMENTS}

This work was supported by grants R01 DC004554 to T.R. and R01 DC02368 to P.G. from the National Institute of Deafness and Other Communication Disorders, National Institutes of Health.

\section{REFERENCES}

Aibara R, Welsh JT, Puria S, Goode RL (2001) Human middle-ear sound transfer function and cochlear input impedance. Hear Res 152:100-109

Cooper NP, RHode WS (1992) Basilar membrane mechanics in the hook region of cat and guinea-pig cochleae: sharp tuning and nonlinearity in the absence of baseline position shifts. Hear Res 63:163-190

DE Boer E (1996) Mechanics of the cochlea: modeling efforts. In: Dallos P, Popper AN, Fay RR (eds) The cochlea. Springer, New York, pp 258-317

De Boer E, Nuttall AL (1999) The “inverse problem" solved for a three-dimensional model of the cochlea. III. Brushing-up the solution method. J Acoust Soc Am 105:3410-3420 
Dong W, Olson ES (2008) Supporting evidence for reverse cochlear traveling waves. J Acoust Soc Am 123:222-240

Lighthill J (1981) Energy flow in the cochlea. J Fluid Mech 106:149-213

Lynch TJ 3Rd, Nedzelnitsky V, Peake WT (1982) Input impedance of the cochlea in cat. J Acoust Soc Am 72:108-130

Nuttall AL, Dolan DF, Avinash G (1991) Laser Doppler velocimetry of basilar membrane vibration. Hear Res 51:203-213

OLSON ES (2001) Intracochlear pressure measurements related to cochlear tuning. J Acoust Soc Am 110:349-367

OLSON ES (2004) Harmonic distortion in intracochlear pressure and its analysis to explore the cochlear amplifier. J Acoust Soc Am 115:1230-1241

Peterson LC, Bogert BP (1950) A dynamic theory of the cochlea. J Acoust Soc Am 22:369-381

PURIA S (2003) Measurements of human middle ear forward and reverse acoustics: implications for otoacoustic emissions. J Acoust Soc Am 113:2773-2789

Puria S, Steele C (2008) Mechano-acoustical transformations. In: Dallos P, Oertel D (eds) A comprehensive reference, vol 3, Audition. Academic, San Diego, pp 165-202

REN T (2002) Longitudinal pattern of basilar membrane vibration in the sensitive cochlea. Proc Natl Acad Sci USA 99:17101-17106
Ren T, He W, Gillespie PG (2011A) Measurement of cochlear power gain in the sensitive gerbil ear. Nat Commun 2:216-223

Ren T, He W, Porsov E (2011B) Localization of the cochlear amplifier in living sensitive ears. PLoS One 6:e20149

RHoDE WS (1971) Observations of the vibration of the basilar membrane in squirrel monkeys using the Mossbauer technique. J Acoust Soc Am 49:1218-1231

Robles L, Ruggero MA (2001) Mechanics of the mammalian cochlea. Physiol Rev 81:1305-1352

Ruggero MA, Rich NC (1991) Application of a commerciallymanufactured Doppler-shift laser velocimeter to the measurement of basilar-membrane vibration. Hear Res 51:215-230

Russell IJ, NiLSEn KE (1997) The location of the cochlear amplifier: spatial representation of a single tone on the guinea pig basilar membrane. Proc Natl Acad Sci USA 94:2660-2664

Shera CA, Olson ES, Guinan JJ, Jr. (2011) On cochlear impedances and the miscomputation of power gain.J Assoc Res Otolaryngol (in press)

Wever EG, LaWrence M, von BeKesy G (1954) A note on recent developments in auditory theory. Proc Natl Acad Sci USA 40:508-512

ZweIg G (1976) Basilar membrane motion. Cold Spring Harb Symp Quant Biol 40:619-633

ZWISLOCKI JJ (1953) Wave motion in the cochlea caused by bone conduction. J Acoust Soc Am 25:986-989 